\title{
Ethyl Acetate Fraction of Adenophora triphylla var. japonica Inhibits Migration of Lewis Lung Carcinoma Cells by Suppressing Macrophage Polarization toward an M2 Phenotype
}

\author{
Shin-Hyung Park ${ }^{1 *}$ \\ ${ }^{1}$ Departments of Pathology, College of Korean Medicine, Dong-eui University, Busan, 47227, Republic of Korea
}

\begin{abstract}
Key Words
Adenophora triphylla var. japonica, M2 macrophage, Macrophage polarization, Tumor-associated macrophage, Tumor microenvironment, Lung cancer, Migration
\end{abstract}

\begin{abstract}
Objectives: It is reported that tumor-associated macrophages (TAMs) contribute to cancer progression by promoting tumor growth and metastasis. The purpose of this study is to investigate the effect of different fractions of Adenophora triphylla var. japonica (AT) on the polarization of macrophages into the M2 phenotype, a major phenotype of TAMs.
\end{abstract}

Methods: We isolated hexane, ethyl acetate, and butanol fractions from crude ethanol extract of AT. The cytotoxicity of AT in RAW264.7 cells was examined by 3-(4,5-Dimethylthiazol-2-yl)-2,5-diphenyltetrazolium bromide (MTT) assay. RAW264.7 cells were polarized into the M2 phenotype by treatment with interleukin (IL)-4 and IL-13. The expression of M2 macrophage marker genes was detected by reverse transcription polymerase chain reaction (RT-PCR). The phosphorylation level of signal transducer and activator of transcription 6 (STAT6) was investigated by western blot analysis. The migration of Lewis lung carcinoma (LLC) cells was examined by transwell migration assay using conditioned

Received: Aug 21, 2019 Reviewed: Nov 27, $2019 \quad$ Accepted: Nov 27, 2019

(a) This is an Open-Access article distributed under the terms of the Creative Commons Attribution Non-Commercial License (http://creativecommons.org/licenses/by-nc/4.0/) which permits unrestricted noncommercial use, distribution, and reproduction in any medium, provided the original work is properly cited.

(2) This paper meets the requirements of KS X ISO 9706, ISO 9706-1994 and ANSI/NISO Z39.48-1992 (Permanence of Paper). media (CM) collected from RAW264.7 cells as a chemoattractant.

Results: Among various fractions of AT, the ethyl acetate fraction of AT (EAT) showed the most significant suppressive effect on the mRNA expression of M2 macrophage markers, including arginase-1, interleukin (IL)-10 and mannose receptor C type 1 (MRC-1), up-regulated by treatment of IL-4 and IL-13. In addition, EAT suppressed the phosphorylation of STAT6, a critical regulator of IL-4 and IL-13-induced M2 macrophage polarization. Finally, the increased migration of Lewis lung carcinoma (LLC) cells by CM from M2-polarized RAW264.7 cells was reduced by CM from RAW264.7 cells co-treated with EAT and M2 polarization inducers.

Conclusion: We demonstrated that EAT attenuated cancer cell migration through suppression of macrophage polarization toward the M2 phenotype. Additional preclinical or clinical researches are needed to evaluate its regulatory effects on macrophage polarization and anti-cancer activities.

\section{Introduction}

Tumor microenvironment (TME) is the environment around a tumor, composed by various stromal cells including endothelial cells, immune cells, fibroblasts, and the extracellular matrix. In the past decade, the importance of TME on tumor progression has risen in prominence. The stromal cells in tumors significantly influence most of hallmarks of cancers such as sus-

"Corresponding Author

Shin-Hyung Park. College of Korean Medicine, Dong-eui University, 52-57 YangJeong-ro, Busanjin-gu, Busan, 47227, Republic of Korea.

Tel: +82-51-850-8646 Fax: +82-51-853-4036

E-mail: omdpark@deu.ac.kr 
taining cell proliferation, inducing angiogenesis, emerging drug resistance, and activating metastasis [1]. Therefore, understanding the complex interactions between cancer cells and stromal cells is important to improve therapeutic strategies for cancer treatment.

Tumor-associated macrophages (TAMs) are a crucial component of TME. TAMs are thought to promote tumor growth, invasion, and metastasis by polarization towards immunosuppressive M2 phenotype [2]. Macrophages may have both anti- and pro-tumor activities by two distinct polarizations including $\mathrm{M} 1$ and $\mathrm{M} 2$ polarization. M1 macrophages promote Thl responses with tumoricidal effects and are activated by lipopolysaccharide (LPS), interferon- $\gamma$, and tumor necrosis factor $\alpha$ (TNF- $\alpha$ ). On the other hand, the M2 macrophages, activated by interleukin (IL)-4, IL-10, and IL-13, induce Th2 responses and closely related with poor prognosis of cancers [3]. Given this background, TAMs have been recognized as an attractive target in cancer therapy. Several signaling pathways, including C-X-C motif chemokine ligand 12 (CXCL12)/C-X-C chemokine receptor type 4 (CXCR4), C-C motif chemokine ligand 2 (CCL2)/C-C chemokine receptor type 2 (CCR2), and colony stimulating factor 1 (CSF1)/CSF1 receptor (CSF1R) axes, are reported to be involved in the influx of TAMs into tumors and M2 polarization. Small molecules or antibodies to block each of these pathways have shown marked improvement in the response of tumors to chemotherapeutic drugs and radiotherapy in a variety of preclinical studies [4].

Adenophora triphylla var. japonica (AT) called as Sa-sam in Korean has been traditionally used in Asian countries as a herbal medicine for controlling lung diseases such as cough, sputum, asthma, and airway inflammatory diseases [5]. According to the traditional theory of Korean medicine, AT mainly support Qi and nourish Yin in lungs. As Qi and Yin deficiency in lung is the basic pathogenesis of lung cancer, AT has been frequently used to treat lung cancer [6-9]. Recently, Lee et al. have reported that extracts of AT exhibited anti-obesity and hypolipidemia effects [10]. Hu et al. have demonstrated that powder of AT possesses antitussive, expectorant, and anti-inflammatory effects [11]. Also, components of AT including saponins, lupeol, lupenone, cycloartenyl acetate, $\beta$-sitosterol, taraxerol, octacosanoic and praeruptorin, were reported to have anti-oxidative, anti-inflammatory, immunomodulatory, and anti-cancer effects [12-19]. Although the previous studies reporting the anti-cancer effects of AT mainly focused on the cancer cell regulation, the influence of AT on the TME regulation has not been explored yet. Given that the extracts and constituents of AT commonly exhibited anti-inflammatory activities by regulating macrophages, we hypothesized that the extracts of AT would influence macrophage polarization. Therefore, the current study investigated the effects of different fractions of AT on macrophage polarization into the M2 phenotype. In addition, we examined whether the TAM-modulatory effects of AT ultimately regulate the migration of cancer cells.

\section{Materials and Methods}

\subsection{Preparation of various fractions from AT}

Dried roots of AT were bought from Nuri Herb Co. Ltd. (YoungCheon, Gyeongsangbuk-do, Korea). AT (200 g) was pulverized into fine powder and extracted for three times with $1.5 \mathrm{~L}$ of $80 \%$ ethanol at room temperature with shaking $(100 \mathrm{rpm})$ for $24 \mathrm{~h}$. The extract was then filtered, concentrated, and lyophilized. The powder was resuspended in distilled water and further fractionated with hexane, ethyl acetate and butanol, in a stepwise manner. The fractions were designated by HAT (hexane fraction of AT), EAT (ethyl acetate fraction of AT), and BAT (butanol fraction of AT). The fractions were concentrated again and lyophilized. The powder was dissolved in dimethyl sulfoxide (DMSO; Amresco, Solon, OH, USA) as a stock solution at $100 \mathrm{mg} / \mathrm{ml}$ for HAT, and at $200 \mathrm{mg} / \mathrm{ml}$ for EAT and BAT.

\subsection{Cell culture}

RAW264.7 mouse macrophage cells were purchased from American Type Culture Collection (ATCC; Rockville, MD, USA), and mouse Lewis lung carcinoma (LLC) cells were a kind gift from Professor Ki-Tae Ha (Busan National University, Republic of Korea). RAW264.7 cells and LLC cells were grown in Dulbecco's Modified Eagle's Medium (DMEM; WelGENE, Daegu, Korea) supplemented with $10 \%$ fetal bovine serum (FBS; WelGENE) and $1 \%$ antibiotics (WelGENE) at $37^{\circ} \mathrm{C}$ in a humidified incubator under $5 \% \mathrm{CO} 2$.

\subsection{Chemicals, reagents and antibodies}

3-(4,5-dimethylthiazol-2-yl)-2,5-diphenyltetrazolium bromide (MTT) was bought from Duchefa (Haarlem, The Netherlands). Recombinant murine IL-4 and IL-13 were obtained from Peprotech (Rocky Hill, NJ, USA). Primary antibodies against phospho- signal transducer and activator of transcription 6 (STAT6), total-STAT6, and actin were purchased from Santa Cruz Biotechnology (Santa Cruz, CA, USA). Anti-rabbit secondary antibody and anti-mouse secondary antibody were purchased from Enzo Life Sciences (Farmingdale, USA) and Bethyl Laboratories (Montgomery, TX), respectively.

\subsection{MTT assay}

RAW264.7 cells ( $5 \times 104$ cells/well) were seeded in 96-well plates and treated with HAT (50-200 $\mu \mathrm{g} / \mathrm{ml})$, EAT (50-200 $\mu \mathrm{g} / \mathrm{ml})$, and BAT $(50-200 \mu \mathrm{g} / \mathrm{ml})$ for $24 \mathrm{~h}$. Then MTT solution was added to the culture media at final concentration of $0.4 \mathrm{mg} / \mathrm{ml}$. After incubation for $2 \mathrm{~h}$ at $37^{\circ} \mathrm{C}$, the media were discarded and $100 \mu \mathrm{l}$ of DMSO were added to each well to dissolve the formazan. The absorbance was measured using a microplate reader (SpectraMax M3; Molecular Devices, Sunnyvale, CA, USA) at $540 \mathrm{~nm}$.

\subsection{Transwell migration assay}


Transwell migration assay was performed using 24-well transwell with $8.0 \mu \mathrm{m}$ pore size (Corning, NY, USA)). The outer membrane of upper well was coated with $0.1 \%$ gelatin (Sciencell, Carlsbad, CA, USA). In order to investigate the migration ability of LLC cells, LLC cells $(2 \times 105$ cells) suspended in serum-free media were seeded onto the upper wells and conditioned media (CM) from RAW264.7 cells were used as a chemoattractant in down chambers. After $24 \mathrm{~h}$ of incubation, the membrane was stained with hematoxylin solution (Sigma-Aldrich, St. Louis, MO, USA). The stained cells were photographed at $\times 100$ magnification using a microscope and the number of stained cells per field was counted. In order to collect CM from RAW264.7, RAW264.7 cells were treated with IL- 4 and IL-13 w/ or w/o EAT for $24 \mathrm{~h}$, and the media was replaced with fresh serum free media. After 24 $\mathrm{h}$, the culture media was collected from RAW264.7 cells.

\subsection{Reverse transcription polymerase chain reaction (RT-PCR)}

Cells were harvested and total RNA was extracted using TRIzol reagent (Invitrogen, Carlsbad, CA, USA). RNA was resuspended in $50 \mu \mathrm{l}$ nuclease-free water (QIAGEN, Hilden, Germany), and quantified using a microplate reader (SpectraMax M3). $1 \mu \mathrm{g}$ of RNA was used to synthesize cDNA using PrimeScript RT reagent kit (Takara, Tokyo, Japan) according to the manufacturer's instruction. The primer sequences used are as follows: mouse arginase-1 forward, 5'-AAC CAG CTC TGG GAA TCT GC-3' and reverse, 5'- TCC ATC ACC TTG CCA ATC CC-3'; mouse mannose receptor $C$ type $1(M R C$ - 1$)$ forward, 5 '-TTC GGG ATT GTG GAG CAG ATC-3' and reverse, 5'-TTG TCG TAG TCA GTG GTG GTT C-3'; mouse IL-10 forward, 5'-CTC TTA CTG ACT GGC ATG AGG AT-3' and reverse, 5'-GAG TCG GTT AGC AGT ATG TTG T-3'; and mouse glyceraldehyde-3-phosphate dehydrogenase (GAPDH) forward, 5'-AAC TTT GGC ATT GTG GAA GG-3' and reverse, 5'-ACA CAT TGG GGG TAG GAA CA-3'. cDNA was amplified using a SimpliAmp Thermal Cycler (Applied Biosystems, Forster City, CA, USA). Cycle numbers were determined to be 30 cycles at an annealing temperature of $55^{\circ} \mathrm{C}$ for arginase-1, 35 cycles at $53^{\circ} \mathrm{C}$ for $M R C-1,32$ cycles at $55^{\circ} \mathrm{C}$ for IL-10, and 20 cycles at $55^{\circ} \mathrm{C}$ for $G A P D H$. The PCR products were loaded on a $1.5 \%$ agarose gel (Lonza, Walkersville, MD, USA) containing a nucleic acid staining solution (iNtRON Biotechnology, Seongnam, Korea) for electrophoresis, and visualized by the Gel Imaging System (Daihan Scientific, Seoul, Korea).

\subsection{Western blot}

Cells were scraped off and lysed by freezing and thawing in cold radio-immunoprecipitation assay (RIPA) buffer (Thermo Scientific, Waltham, MA, USA) supplemented with a protease inhibitor cocktail (Thermo Scientific) and phosphatase inhibitors (1 mM Na3VO4 and $100 \mathrm{mM} \mathrm{NaF}$ ), and incubated at $4^{\circ} \mathrm{C}$ for $30 \mathrm{~min}$. After quantification of proteins using a bicinoconinic acid (BCA) protein assay kit (Pierce Biotechnology, Rockford, IL, USA), the same amounts of proteins were separated by sodium dodecyl sulfate (SDS)-polyacrylamide gels and transferred onto a polyvinyl difluoride (PVDF) membrane (Millipore, Bedford, MA, USA). Then the membrane was blocked with $3 \%$ bovine serum albumin (BSA, GenDEPOT, TX, USA) for 30 min at room temperature. Target proteins were probed by primary antibodies for overnight at $4^{\circ} \mathrm{C}$, and consequently by respective secondary antibodies for $1 \mathrm{~h}$ at room temperature. The protein expression was detected by D-Plus ECL Femto System (Donginbio, Seoul, Korea) as described in the manufacturer's recommended protocol.

\subsection{Statistical analysis}

Each result presented is shown as mean \pm SD of three or more independent experiments. Statistical significance was estimated by Student's t-test. Differences were considered significant at $P<0.05$.

\section{Results}

\subsection{Effects of different fractions of AT on the expression of M2 markers in RAW264.7 cells}

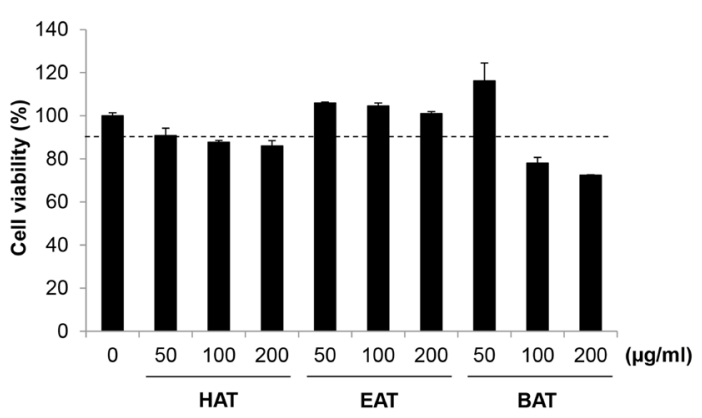

Figure 1 Effects of the AT subfractions on the cell viability in RAW264.7 cells

RAW264.7 mouse macrophage cells were treated with the three subfractions of AT for $24 \mathrm{~h}$. The cell viability was evaluated by MTT assay. Data are expressed as the mean \pm S.D. of three independent experiments. AT, Adenophora triphylla var. japonica; HAT, hexane fraction of AT; EAT, ethyl acetate fraction of AT; BAT, butanol fraction of AT.

In order to determine the concentrations of three fractions of AT that have no cytotoxicity in RAW264.7 cells, MTT assay was conducted. As shown in Figure 1, the maximum concentration with cell viability more than $90 \%$ was $50 \mu \mathrm{g} / \mathrm{mL}$ for hexane fraction (HAT) and butanol fraction (BAT), and $200 \mu \mathrm{g} / \mathrm{mL}$ for ethyl acetate fraction (EAT) (Figure 1). Thus, we used $50 \mu \mathrm{g} / \mathrm{mL}$ and $200 \mu \mathrm{g} / \mathrm{mL}$ as the maximum concentration of HAT/BAT and EAT, respectively, 
A)

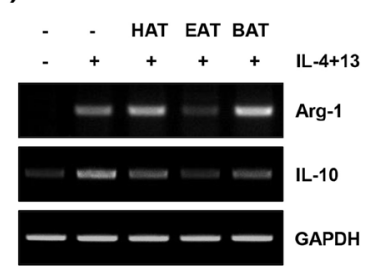

B)

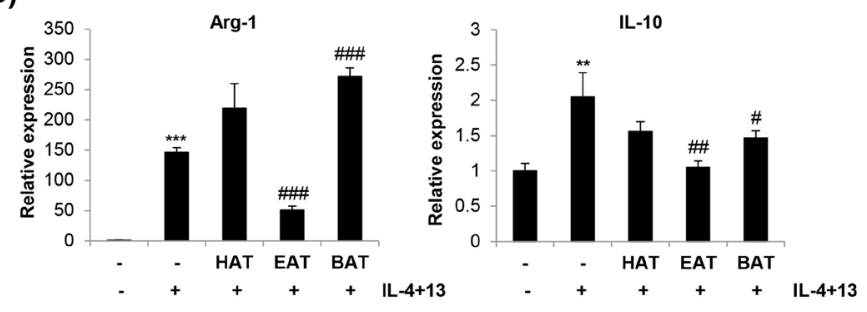

Figure 2 Effects of the AT subfractions on the expression of M2 marker genes in RAW264.7 cells

RAW264.7 mouse macrophage cells were treated with IL-4 $(20 \mathrm{ng} / \mathrm{ml})$ and IL-13 $(20 \mathrm{ng} / \mathrm{ml})$ to stimulate M2 polarization, and challenged with the three subfractions of AT ( $50 \mu \mathrm{g} / \mathrm{ml}$ for HAT and BAT, $200 \mu \mathrm{g} / \mathrm{ml}$ for EAT) for $24 \mathrm{~h}$. The expression of M2 marker genes including arginase-1 and IL-10 was detected by RT-PCR. (A) Representative images of three independent experiments were shown. (B) Relative expression of arginase-1 and IL-10 compared with the untreated cells was calculated using Image J software. Actin was used for normalization. Data are expressed as the mean \pm S.D. of three independent experiments. Significance was determined by the Student's t-test $\left({ }^{* *} P<0.01\right.$, ${ }^{* * *} P<0.001$ vs. untreated control; \#P<0.05,\# $P<0.01$, \#\# $P<0.001$ vs. IL-4 and IL-13-treated cells). AT, Adenophora triphylla var. japonica; HAT, hexane fraction of AT; EAT, ethyl acetate fraction of AT; BAT, butanol fraction of AT; Arg-1, arginase-1; IL-10, interleukin-10.

A)

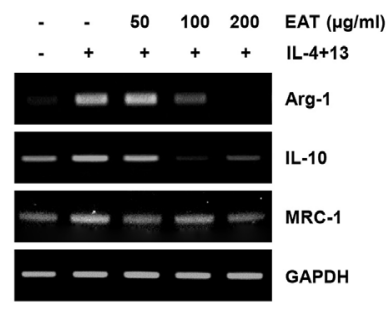

B)

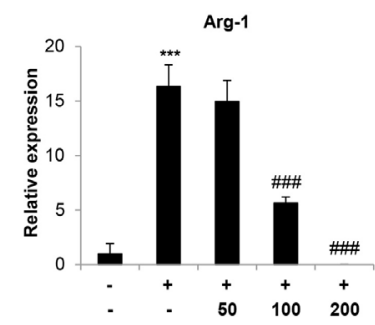

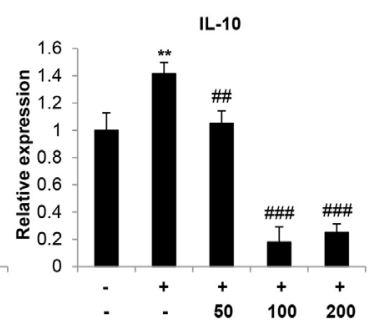

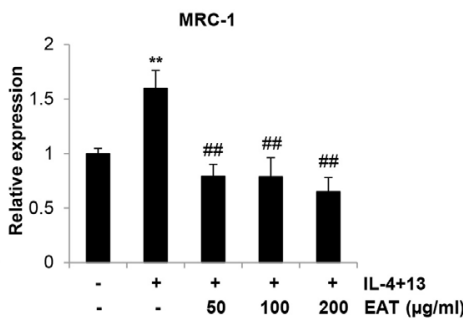

Figure 3 Effects of EAT on the expression of M2 marker genes in RAW264.7 cells

RAW264.7 mouse macrophage cells were treated with IL-4 $(20 \mathrm{ng} / \mathrm{ml})$ and IL-13 $(20 \mathrm{ng} / \mathrm{ml})$ to stimulate M2 polarization, and challenged with the indicated concentrations of EAT. The expression of M2 marker genes including arginase-1, $I L-10$, and $M R C-1$ was detected by RT-PCR. (A) Representative images of three independent experiments were shown. (B) Relative expression of the indicated genes compared with the untreated cells was calculated using Image J software. Actin was used for normalization. Data are expressed as the mean \pm S.D. of three independent experiments. Significance was determined by the Student's t-test ${ }^{* *} P<0.01$, ${ }^{* * *} P<0.001$ vs. untreated control; \#\# $P<0.01$, \#\#\# $P<0.001$ vs. IL-4 and IL-13-treated cells). EAT, ethyl acetate fraction of Adenophora triphylla var. japonica; Arg-1, arginase-1; IL-10, interleukin-10; MRC-1, mannose receptor C type 1.

A)

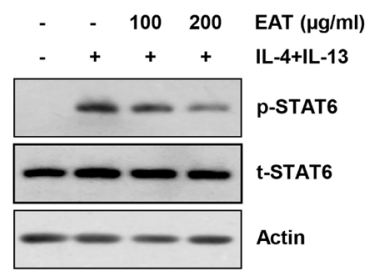

B)

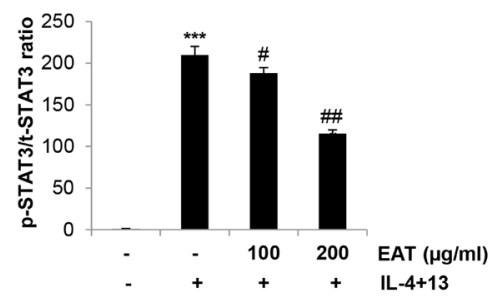

Figure 4 Effects of EAT on the phosphorylation level of STAT6 in RAW264.7 cells

RAW264.7 mouse macrophage cells were treated with IL-4 $(20 \mathrm{ng} / \mathrm{ml})$ and IL-13 $(20 \mathrm{ng} / \mathrm{ml})$ w/ or w/o EAT for 24 h. The cell lysates were subjected for western blot analysis. The expression of phosphorylated STAT6 (p-STAT6) and total STAT6 (t-STAT6) was evaluated. (A) Representative images of three independent experiments were shown. (B) The ratio of p-STAT6/t-STAT6 was calculated using Image J software after normalization with actin. Data are expressed as the mean \pm S.D. of three independent experiments. Significance was determined by the Student's t-test (*** $\mathrm{P}<0.001$ vs. untreated control; \# $\mathrm{P}<0.05$, \#\# $\mathrm{P}<0.01$ vs. IL-4 and IL-13-treated cells). EAT, ethyl acetate fraction of Adenophora triphylla var. japonica 
for further experiments.

Next, we investigated the modulatory effects of three fractions of AT on M2 polarization of macrophages. We examined the mRNA expression of M2 marker genes including arginase- 1 and $I L-10$ following treatment with AT subfractions. Compared with the other fractions, EAT exhibited the strongest suppressive effect on the expression of M2 markers stimulated by treatment with IL-4 and IL-13 (Figure $2 \mathrm{~A}$ and $2 \mathrm{~B}$ ). RAW264.7 cells treated with HAT or BAT showed no or just a slight decrease in the expression of arginase- 1 and $I L-10$ (Figure $2 \mathrm{~A}$ and $2 \mathrm{~B}$ ). Therefore, we further investigated the concentration-dependent regulation of EAT on the expression of M2 markers in RAW264.7 cells. The results showed that EAT treatment down-regulated the mRNA expression of arginase-1, $I L-10$, and $M R C-1$ in a concentration-dependent manner even though IL-10 level was slightly recovered at $200 \mu \mathrm{g} / \mathrm{mL}$ (Figure 3A and $3 B$ ). These results clearly suggest that EAT suppressed the macrophage polarization toward the M2 phenotype.

\subsection{Effects of EAT on STAT6 phosphoryla- tion in RAW264.7 cells}

We next investigated whether EAT inhibits STAT6 phosphorylation in RAW264.7 cells. When IL-4 or IL-13 combines with the receptors, STAT6 is phosphorylated and stimulates the transcription of M2 marker genes such as arginase-1, $I L-10, M R C-1$, found in the inflammatory zone 1 (Fizzl) and chitinase 3-like 3 lectin (Ym1) [20]. In the tumor microenvironment, IL- 4 and IL-13 are secreted from cancer cells and shift the macrophages infiltrated in tumors into TAMs which resemble M2 macrophages [21]. Our results showed that EAT inhibited the IL-4 and IL-13induced phosphorylation of STAT6 in a concentration-dependent manner (Figure 4A and 4B). These results suggest that EAT suppressed M2 polarization of RAW264.7 cells by regulating STAT6 activity.

\subsection{Effects of EAT on the migration of Lew- is lung carcinoma cells}

Previous studies have reported that TAMs promote migration and metastasis of cancers by secreting various soluble factors [2]. Because we already observed that EAT suppressed the M2 polarization, we hypothesized that EAT-regulated macrophage polarization would affect to the migration ability of cancer cells. To verify our hypothesis, transwell migration assay was conducted. We used CM from RAW264.7 cells as a chemoattractant in down chambers and loaded LLC cells onto upper chambers to mimic co-culture between LLC cells and RAW264.7 cells, and to exclude the direct influence of IL-4/IL-13 and EAT on LLC cells (Figure 5A). After $24 \mathrm{~h}$ of incubation, migration of LLC cells increased when CM from M2-polarized RAW264.7 cells was used as a chemoattractant (Figure 5B and 5C). However, when CM from RAW264.7 cells co-treated with EAT and M2 polarization inducers was loaded in down chambers, the migration of LLC cells was significantly
A)

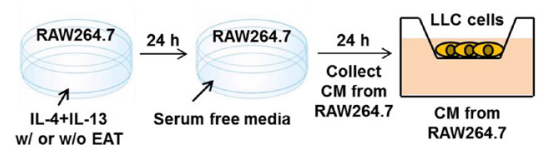

B)

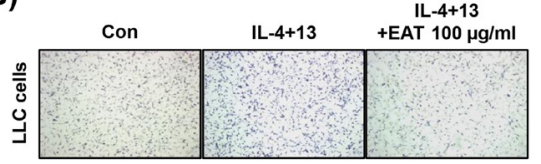

C)

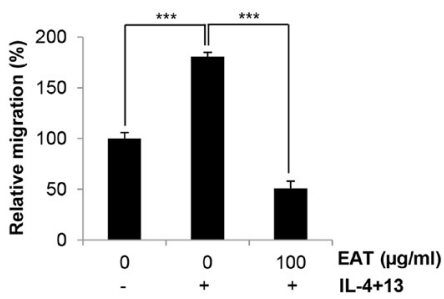

Figure 5 Effects of EAT on the migration of Lewis lung carcinoma cells

(A) Experimental scheme of transwell migration assay is shown. RAW264.7 cells were treated with IL-4 (20 ng/ml) and IL-13 (20 $\mathrm{ng} / \mathrm{ml}$ ) w/ or w/o EAT for $24 \mathrm{~h}$. The culture media was then replaced with fresh serum free media. After $24 \mathrm{~h}$ of incubation, the conditioned media was collected from RAW264.7 cells and filled in the bottom chamber of 24-well transwell plate as a chemoattractant. Lewis lung carcinoma cells were plated in triplicate into the upper chamber and incubated for $24 \mathrm{~h}$. (B) Cells that migrated through the membrane were photographed by a microscope ( $\times 100$ magnification). (C) The migrated cells were counted and the relative migration was calculated compared with the control cells. Data are expressed as the mean \pm S.D. of three independent experiments. Significance was determined by the Student's t-test (*** $P<0.001$ vs. respective control). CM, conditioned media; LLC, Lewis lung carcinoma; EAT, ethyl acetate fraction of Adenophora triphylla var. japonica. 
reduced (Figure 5B and 5C). These results clearly demonstrate that EAT blocked cancer cell migration by suppressing M2 polarization of macrophages.

\section{Discussion}

The present study explored the modulatory effects of AT subfractions on the macrophage polarization into the M2 phenotype. We found that EAT exhibited the strongest inhibitory effects on M2 polarization in RAW264.7 cells. EAT downregulated the expression of M2 marker genes as well as the phosphorylation of STAT6, both of which increased by treatment with IL-4 and IL-13. Given that STAT6 mediates IL-4 or IL-13-induced M2 polarization of macrophages $[20,21]$, we suggest that EAT inhibited the M2 polarization of RAW264.7 cells by suppression of STAT6 activity. In addition, we found that the suppression of M2 polarization by EAT affected to the migration ability of lung cancer cells. These results collectively demonstrate that EAT possesses anticancer effects by modulation of TAMs.

It is reported that several components of AT including lupenone, lupeol, taraxerol, and erandone showed anti-inflammatory effects [12-17]. In the current study, we demonstrated that EAT attenuated macrophage polarization into the immunosuppressive M2 phenotype. Because both severe inflammation and immune suppression are associated with the development and progression of cancer $[22,23]$, these conflicting role of AT on the regulation of immune system suggests that the immune dysfunction in cancer could be normalized by EAT. As regards direct anticancer activities, $\beta$-sitosterol isolated from AT inhibited invasion and metastasis of tumor cells [17]. In addition, saponins isolated from AT suppressed cancer cell proliferation by apoptosis induction $[18,19]$. Although the current study did not determine the specific compound of EAT which regulates TAMs, our results and the previous studies collectively suggest that AT could be a desirable candidate for treatment of cancers. Given that herbal medicines generally have multiple compounds and various mechanisms of action, it can be worth exploring whether the active compounds of AT exhibits synergistic anticancer effects.

It is still needed to determine the precise molecular mechanism of EAT-suppressed cancer cell migration. Generally, CXCL12/CXCR4, CCL2/CCR2, and CSF1/CSF1R signaling pathways are reported to be involved in the crosstalk between cancer cells and TAMs [4]. For example, Choi et al. have reported that luteolin suppressed the migration of lung cancer cells by blocking CCL2 secretion from M2 macrophages [24]. In addition, Ding et al. have shown that M2 macrophages stimulated angiogenesis and cancer cell migration by secreting various factors including fibroblast growth factor 1 (FGF1), CCL2, CCL7 and CXCL2, which was abrogated by metformin treatment [25]. Therefore, it is required to further identify the specific secreted factor regulated by EAT treatment in RAW264.7 cells for understating the molecular mechanism and the application of targeted therapies which raise the anticancer effects of EAT. In conclusion, our study demonstrated that EAT suppressed the macrophage polarization towards the M2 phenotype, leading to the attenuation of cancer cell migration.
We provide a valuable information for finding new anticancer materials from traditional herbal medicines based on their TAM-modulatory effects. We suggest that AT could be a novel therapeutic candidate for treatment of cancers.

\section{Acknowledgement}

This study was supported by grants from the National Research Foundation of Korea (NRF), Republic of Korea (No. NRF-2016R1C1B2015076), and by grants from the Dongeui University Grant (No. 201902120001), Republic of Korea.

\section{Declaration of conflicts of interest}

There are no conflicts of interest.

\section{Conflict of interest}

We have no conflict of interest to declare.

\section{References}

1. Hanahan D, Coussens LM. Accessories to the crime: functions of cells recruited to the tumor microenvironment. Cancer Cell. 2012;21(3):309-22.

2. J.W. Pollard. Tumour-educated macrophages promote tumour progression and metastasis. Nat Rev Cancer. 2004;4(1):71-8.

3. Mei J, Xiao Z, Guo C, Pu Q, Ma L, Liu C, et al. Prognostic impact of tumor-associated macrophage infiltration in non-small cell lung cancer: A systemic review and meta-analysis. Oncotarget. 2016;7(23):34217-28.

4. Brown JM, Recht L, Strober S. The Promise of Targeting Macrophages in Cancer Therapy. Clin Cancer Res. 2017;23(13):3241-50.

5. Jang IM. [Treatise on Asian herbal medicines]. Seoul: Haksul-pyunsu-kwan in Research Institute of Natural Products of Seoul National University; 2003. Korean.

6. Geng G, Sun FY. Efficacy of Yiqi Yangyin in the treatment of advanced non-small cell lung cancer. Nei Mongol Journal of Traditional Chinese Medicine. 1991;4:45.

7. Ma CY, Tang YJ. Application of Yiqi Yangyin in lung cancer. Journal of Shandong college of traditional chinese medicine. 1996;20(3):178-9.

8. Zhang T, Wang B. Clinical study on integrated Chinese and western medicine combined with radiotherapy in treatment of patients with advanced lung cancer. Journal of Clinical and Experimental Medicine. 2013;12(2):108-10.

9. Liang F, Zhang Y, Li CH, Cao HT. Effect of Yiqi Yangyin Jiedu Formula on T-Cell Subsets and Shifting of Thl/ Th2 Balance in NSCLC Patients. Journal of Practical Traditional Chinese Internal Medicine. 2011;25(5):810. 
10. Lee DR, Lee YS, Choi BK, Lee HJ, Park SB, Kim TM, et al. Roots extracts of Adenophora triphylla var. japonica improve obesity in 3T3-L1 adipocytes and highfat diet-induced obese mice. Asian Pac J Trop Med. 2015;8(11):898-906.

11. Hu JR, Jung CJ, Ku SM, Jung DH, Ku SK, Choi JS. Antitussive, expectorant, and anti-inflammatory effects of Adenophorae Radix powder in ICR mice. J Ethnopharmacol. 2019;239:111915.

12. Jin SE, Son YK, Min BS, Jung HA, Choi JS. Anti-inflammatory and antioxidant activities of constituents isolated from Pueraria lobata roots. Arch Pharm Res. 2012;35(5):823-37.

13. Kang SC, Lim SY, Song YJ. Lupeol is one of active components in the extract of Chrysanthemum indicum Linne that inhibits LMP1-induced NF-kappaB activation. PLoS One. 2013;8(11):e82688.

14. Yao X, Li G, Bai Q, Xu H, Lu C. Taraxerol inhibits LPS-induced inflammatory responses through suppression of TAK1 and Akt activation. Int Immunopharmacol. 2013;15(2):316-24.

15. Srivastava P, Jyotshna, Gupta N, Maurya AK, Shanker K. New anti-inflammatory triterpene from the root of Ricinus communis. Nat Prod Res. 2014;28(5):306-11.

16. Yoon YP, Lee HJ, Lee DU, Lee SK, Hong JH, Lee CJ. Effects of Lupenone, Lupeol, and Taraxerol Derived from Adenophora triphylla on the Gene Expression and Production of Airway MUC5AC Mucin. Tuberc Respir Dis (Seoul). 2015;78(3):210-7.

17. Ovesna Z, Vachalkova A, Horvathova K. Taraxasterol and beta-sitosterol: new naturally compounds with chemoprotective/chemopreventive effects. Neoplasma. 2004;51(6):407-14.

18. Chun J, Kang M, Kim YS. A triterpenoid saponin from Adenophora triphylla var. japonica suppresses the growth of human gastric cancer cells via regulation of apoptosis and autophagy. Tumour Biol. 2014;35(12):12021-30.

19. Kang M, Ha IJ, Chun J, Kang SS, Kim YS. Separation of two cytotoxic saponins from the roots of Adenophora triphylla var. japonica by high-speed counter-current chromatography. Phytochem Anal. 2013;24(2):148-54.

20. Bi Y, Chen J, Hu F, Liu J, Li M, Zhao L. M2 Macrophages as a Potential Target for Antiatherosclerosis Treatment. Neural Plast. 2019;2019:6724903.

21. G Solinas, G Germano, A Mantovani, P Allavena. Tumor-associated macrophages (TAM) as major players of the cancer-related inflammation. J Leukoc Biol. 2009;86(5):1065-73.

22. Singh N, Baby D, Rajguru JP, Patil PB, Thakkannavar SS, Pujari VB. Inflammation and cancer. Ann Afr Med. 2019;18(3):121-6.

23. Finn OJ. Immuno-oncology: understanding the function and dysfunction of the immune system in cancer. Ann Oncol. 2012;23(Suppl 8):viii6-9.

24. Choi HJ, Choi HJ, Chung TW, Ha KT. Luteolin inhibits recruitment of monocytes and migration of Lewis lung carcinoma cells by suppressing chemokine (C-C motif) ligand 2 expression in tumor-associated macrophage. Biochem Biophys Res Commun. 2016;470(1):101-6.

25. Oncotarget. 2015 Nov 3;6(34):36441-55. doi: 10.18632/ oncotarget.5541. Metformin prevents cancer metastasis by inhibiting M2-like polarization of tumor associated macrophages. Ding L1, Liang G1, Yao Z1, Zhang J1, Liu R1, Chen H1, Zhou Y1, Wu H1, Yang B1, He Q1. 